\title{
Nonspecific Interstitial Pneumonia with Abundant IgG4-positive Cells Infiltration, Which was Thought as Pulmonary Involvement of IgG4-related Autoimmune Disease
}

\author{
Hazuki Takato ${ }^{1}$, Masahide Yasui ${ }^{1}$, Yukari Ichikawa ${ }^{1}$, Masaki Fujimura ${ }^{1}$, Shinji Nakao ${ }^{1}$, \\ Yoh Zen ${ }^{2}$ and Hiroshi Minato ${ }^{3}$
}

\begin{abstract}
Recently, great attention has been drawn to IgG4-related diseases such as autoimmune pancreatitis (AIP) sclerosing sialadenitis, retroperitoneum fibrosis, sclerosing cholangitis. IgG4-related diseases are characterized by high serum IgG4 concentrations, sclerosing inflammation with numerous IgG4-positive plasma cells, and steroid sensitivity irrespective of their organs of origin. In this report, we describe a case of nonspecific interstitial pneumonia, in which possible involvement of IgG4 was suggested. The patient was 59-year-old man, who was found to have bilateral interstitial pneumonia. Laboratory tests revealed that he had antinuclear antibody and a high serum IgG4 concentration. Pathological examination of the video-assisted thoracic surgery biopsy taken from the right lower lobe showed interstitial thickening associated with lymphoplasmacytic infiltration containing many IgG4-positive plasma cells. He was effectively treated by corticosteroid. The present case had many clinical and clinicopathologic similarities to systemic IgG4-related autoimmune disease. There have been no descriptions on isolated interstitial pneumonia with IgG4-positive plasma cell infiltration. This case suggested that IgG4-related disorders could also occur in the lung, and interstitial pneumonia may be a pulmonary manifestation of systemic IgG4-related autoimmune disease.
\end{abstract}

Key words: autoimmune diseases, IgG4, interstitial lung diseases, lung fibrosis

(DOI: 10.2169/internalmedicine.47.0411)

\section{Introduction}

IgG4-positive plasma cell infiltration occurs in almost all major organs, including the lung. Some of these IgG4related disease are known as autoimmune pancreatitis (AIP) sclerosing sialadenitis, sclerosing cholangitis. These conditions share clinical and pathological characteristics such as high serum IgG4 concentrations, sclerosing inflammation with many IgG4-positive plasma cells, and effectiveness of corticosteroid therapy irrespective of their organ of origin. As for pulmonary involvement of IgG4-related disease, inflammatory pseudotumor (plasma cell granuloma) of the lung (1), or interstitial pneumonia associated with AIP (2) are known.

\section{Case Report}

A 59-year-old man presented with a 3-month history of dry cough and shortness of breath upon exertion. He had a history of heavy smoking and was a current smoker (40 cigarettes/day for 40 years). He worked as a truck driver hauling sand or stone, and had a history of occupational inhalation of stone dust. He visited Kanazawa University Hospital in December 2004 because his symptoms had not improved. He was found to have bilateral interstitial pneumonia on chest X-ray upon first examination. His cough had decreased after he stopped smoking, but it worsened again

\footnotetext{
${ }^{1}$ Department of Respiratory Medicine, Cellular Transplantation Biology, Kanazawa University Graduate School of Medical Science, Kanazawa, ${ }^{2}$ Department of Human Pathology, Kanazawa University Graduate School of Medical Science, Kanazawa and ${ }^{3}$ Department of Pathology and Laboratory Medicine, Kanazawa Medical University, Kanazawa

Received for publication June 27, 2007; Accepted for publication October 12, 2007

Correspondence to Dr. Masahide Yasui, yasui@med3.m.kanazawa-u.ac.jp
} 


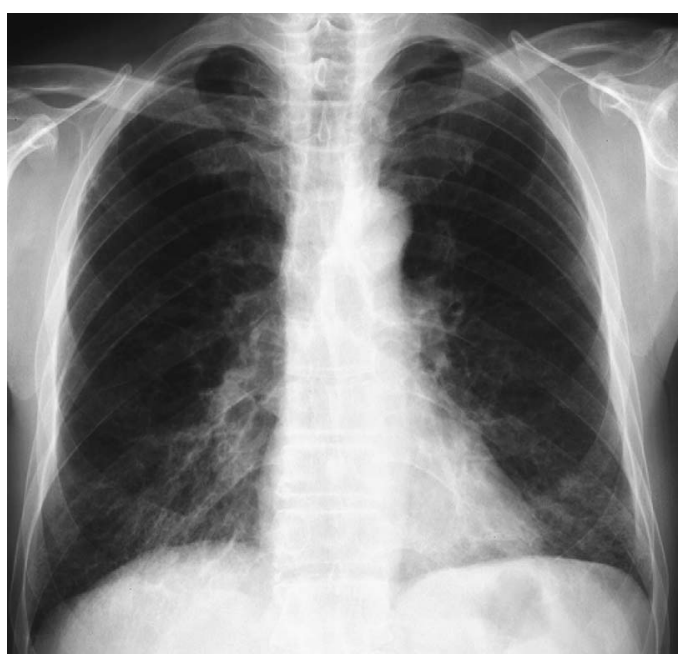

Figure 1. Chest X-ray film obtained upon admission in February 2005 shows volume loss and reticular shadows in both lower lungs.

in February 2005. At approximately the same time, bilateral wrist-hand arthralgia appeared. He was then admitted to our hospital for detailed examination.

Physical examination of the chest on admission revealed bilateral middle-to-late inspiratory fine crackles in the middle and lower zones. And there was clubbing of the extremities. The oxygen saturation was $96 \%$.

The results of respiratory function tests are as follows; a vital capacity (VC) of 3.441 (99.1\% predicted), forced expiratory volume in one second $\left(\mathrm{FEV}_{1}\right)$ of $2.741(100.4 \%$ predicted), residual volume (RV) of 1.861 (107.5\% predicted), total lung capacity (TLC) of 5.301 (97.1\% predicted), RV/ TLC ratio of $35.1 \%$, carbon monoxide diffusing capacity of the lung of $14.02 \mathrm{ml} / \mathrm{min} / \mathrm{mmHg}$ (55.7\% predicted). The carbon monoxide diffusing capacity of the lung was decreased slightly. Arterial blood gas analysis in room air yielded normal values; $\mathrm{pH} 7.43$, arterial oxygen tension $\left(\mathrm{PaO}_{2}\right)$ of $88.5 \mathrm{mmHg}$, arterial carbon dioxide tension $\left(\mathrm{PaCO}_{2}\right)$ of $39.3 \mathrm{mmHg}$. Chest roentgenogram on first examination showed volume loss and reticular shadows in both lower lungs (Fig. 1). Chest CT showed ground-glass opacities and reticular shadows with honeycomb-like changes and traction bronchiectasia, particularly in the lower lobes (Fig. 2). There were no significant findings on abdominal CT.

Peripheral blood examination was as follows: C-reactive protein was $1.1 \mathrm{mg} / \mathrm{dl}$; ESR was $14 \mathrm{~mm} / 1 \mathrm{~h}$. Serum electrolytes, renal function and liver function were normal. Notable laboratory values included: serum KL-6; $793 \mathrm{U} / \mu \mathrm{l}$ (normal: $<500 \mathrm{U} / \mathrm{\mu l}$ ), SP-D; $100 \mathrm{ng} / \mathrm{ml}$ (normal: $<110 \mathrm{ng} / \mathrm{ml}$ ), SP-A; $40.4 \mathrm{ng} / \mathrm{ml}$ (normal: $<43.8 \mathrm{ng} / \mathrm{ml}$ ), TP; $7.2 \mathrm{mg} / \mathrm{dl}$ (alb $49.1 \%, \alpha_{1}$-globlin $3.3 \%, \alpha_{2}$-globlin 9.7\%, $\beta$-globlin $8.8 \%, \gamma-$ globlin $29.1 \%$ ), soluble interleukin-2 receptor, $1,570 \mathrm{U} / \mathrm{ml}$, anti-nuclear antibody was positive $(\times 320$, homogeneous), rheumatoid factor; $14 \mathrm{IU} / \mathrm{ml}, \mathrm{IgG} ; 2,330 \mathrm{mg} / \mathrm{dl}$, IgA; 592 $\mathrm{mg} / \mathrm{dl}$, IgM; $59 \mathrm{mg} / \mathrm{dl}$, IgE; $922 \mathrm{IU} / \mathrm{ml}$; and IgG4 was increased to $325 \mathrm{mg} / \mathrm{dl}, 15.0 \%$ of total $\mathrm{IgG}$ (IgG4 normally

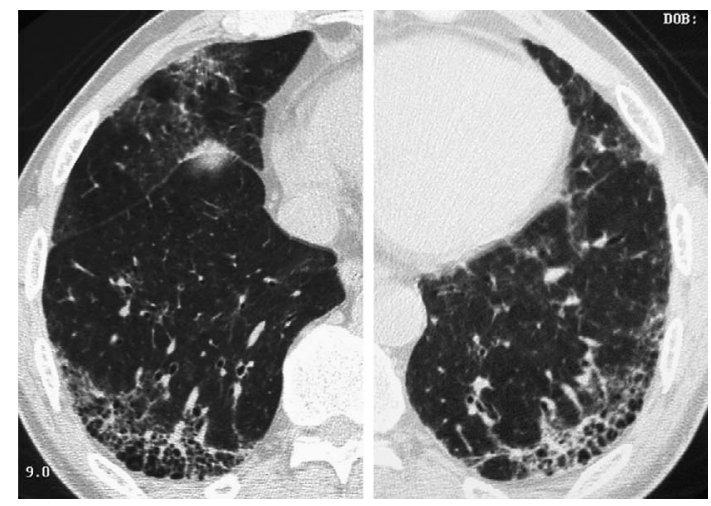

Figure 2. Chest CT scan shows ground-glass opacities and reticular shadows with honeycomb-like changes and traction bronchiectasia especially in the two lower lobes.

comprises less than $6 \%$ of total $\mathrm{IgG})$. Perinuclear antineutrophil cytoplasmic antibody (ab), anti-RNP-ab, antiSSA-ab, anti-SSB-ab, anti-Sm-ab, and anti-centromere-ab, were negative. Aspergillus antigen, aspergillus antibody, candida antigen, and trichosporon antibody were negative.

Bronchoscopy with bronchoalveolar lavage was performed through the right $S_{8}$ bronchus revealed a significant increase in lymphocytes: $5.0 \times 10^{5}$ cells $/ \mathrm{ml}$ (macrophages $23.7 \%$, lymphocytes $70 \%$, neutrophils $1.3 \%$, eosinophils $5 \%$ ). The CD4/ 8 ratio was 0.58 .

Gallium scintigraphy revealed strong uptake in both lower lobes. The results of Saxon test and Shirmer's test were within normal limits. Salivary gland scintigraphy detected no abnormalities. The patient did not meet the criteria for a diagnosis of rheumatoid arthritis. Therefore, we speculated that the patient's symptoms were not due to complications of collagen disease.

To assist with diagnosis and its pathogenesis, videoassisted thoracoscopic surgery biopsy (VATS) of the right lower lung $\left(\mathrm{S}_{10}\right)$ was performed in March 2005. Pathological specimens showed significant diffuse interstitial fibrosis with moderate lymphoplasmacytic infiltration (Fig. 3a, 3b). Fibrosis and inflammation were relatively uniformly distributed. It seems there were honeycomb changes on CT scan, however honeycomb fibrosis, fibroblastic foci were not observed on pathologic findings. Some pathologists examined the VATS sample, and they confirmed the lack of vasculitis.

These histological findings corresponded to non-specific interstitial pneumonia (NSIP), a fibrosing pattern. Immunostaining using a monoclonal antibody to IgG4 (Zymed Laboratory Inc; San Francisco, CA) revealed infiltration in the interstitium of numerous IgG4-positive plasma cells (Fig. 3c).

High-dose prednisolone $(60 \mathrm{mg}, 1 \mathrm{mg} / \mathrm{kg} /$ day $)$ therapy was started, and the patient responded so well that the pulmonary reticular shadows decreased, and the dyspnea on effort improved. Serum KL-6, SP-D, and total IgG decreased gradually. In addition, the IgG4 titer decreased from 325 $\mathrm{mg} / \mathrm{dl}$ to $119 \mathrm{mg} / \mathrm{dl}$. The IgG4 in BALF, which was sampled prior to the start of steroid therapy, was measured, but it 


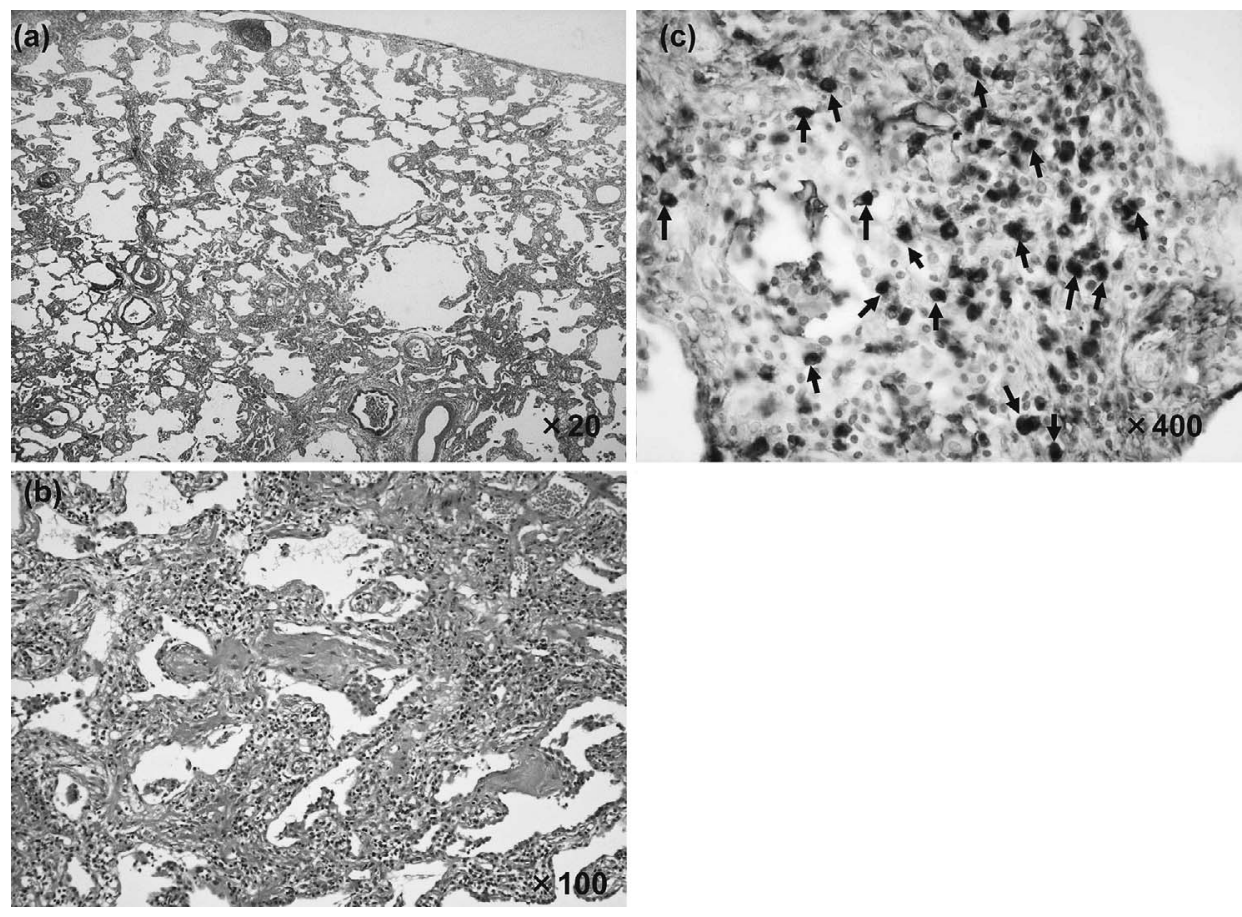

Figure 3. a, b: Histological findings of video-assisted thoracoscopic surgery biopsy specimen from right lower lung. Diffuse alveolar septa thickening and lymphoplasmacytic infiltration were significant in alveolar interstitum. Fibrotic foci were not definite, and a honeycomb structure was not detected (Hematoxylin and Eosin staining, original magnification 3a: $\times 20,3 \mathrm{~b}: \times 100)$. c: Immunostaining of IgG4 in pulmonary tissue. Abundant IgG4-positive plasma cell infiltration (arrows) in the interstitium was detected (original magnification $\times 400$ ).

was under the detection limits of $3.0 \mathrm{mg} / \mathrm{dl}$.

\section{Discussion}

In 2001, IgG4-related disease was first reported with regard to autoimmune pancreatitis (AIP) (3). In that report, patients with autoimmune pancreatitis had significantly higher serum IgG4 concentrations than patients with other pancreas or biliary tract, and they suggested that measuring IgG4 was a useful means of distinguishing this disorder. Later, similar inflammatory conditions characterized by infiltration of many IgG4-positive plasma cells were reported in the bile ducts (sclerosing cholangitis), salivary glands (chronic sclerosing sialadenitis) and retroperitoneum (retroperitoneal fibrosis) $(4,5)$. Some of them synchronously or asynchronously occur in a patient, and they are now thought to be spectrums of a single disease entity. These conditions share clinical and pathological characteristics such as high serum IgG4 concentrations, sclerosing inflammation with many IgG4-positive plasma cells, and effectiveness of corticosteroid therapy irrespective of their organs of origin. The present case also shared these clinicopathological characteristics. In those reports, there was no description about pulmonary lesions involved in IgG4-related disease, but Taniguchi et al reported the first case of interstitial pneumonia associated with IgG4-positive plasma cells, which was found during the follow-up AIP (6). CT findings showed honeycombing of the bilateral lower lung field and ground- glass attenuation in the middle and lower lobes. Hirano et al (2) described four cases of interstitial pneumonia that appeared during follow-up for AIP. AIP preceded the occurrence of interstitial pneumonia in all of these 5 cases. However, in the present case, the absence of association with AIP seems important. Serum IgG4 concentrations or IgG4positive plasma cells have not been examined in patients without AIP to date. And this is the first case of interstitial pneumonia with IgG4-positive plasma cells which is without the complication of AIP. We think IgG4-related interstitial pneumonia unrelated AIP could occur, and it might be included in idiopathic interstitial pneumonia. The correct diagnosis of IgG4-related interstitial pneumonia also seems important because the diagnosis of IgG4-related disease suggests the effectiveness of corticosteroid therapy.

The present case had a history of stone dust inhalation, but the relationship between this episode and the pathogenesis of IgG4-positive plasma cells is obscure. We sent the VATS sample to Niigata University, and the metal components in the lung tissue were analyzed with electro-probe microanalyzer (EPMA). The types of elements which were analyzed were silicon $(\mathrm{Si})$, iron $(\mathrm{Fe})$, titanium (Ti), chromium $(\mathrm{Cr})$, aluminum (Al), tungsten (W). But there was only slight association between pathological lesion and elemental deposit.

\section{Acknowledgement}

We thank Dr. Yoh Zen, Department of Human Pathology, Ka- 
nazawa University Graduate School of Medical Science, for discussion on IgG4 immunostaining. The authors also thank Dr. Mitsuhiro Kawano, Division of Rheumatology and Collagen dis- ease, Kanazawa University Graduate School of Medical Science for providing detailed advice. And we presented this case in the $15^{\text {th }}$ Hakusan Conference (in Kanazawa, January 2006).

\section{References}

1. Zen $\mathrm{Y}$, Kitagawa S, Minato $\mathrm{H}$, et al. IgG4-positive plasma cells in inflammatory pseudotumor (plasma cell granuloma) of the lung. Human Pathology 36: 710-717, 2005.

2. Hirano K, Kawabe T, Komatsu Y, et al. High rate pulmonary involvement in autoimmune pancreatitis. Internal Med J 36: 58-61, 2006

3. Hamano H, Kawa S, Horiuchi A, et al. High serum IgG4 concentrations in patients with sclerosing pancreatitis. N Engl J Med 344: 732-738, 2001.

4. Zen Y, Harada K, Sasaki M, et al. IgG4-related sclerosing chol- angitis with and without hepatic inflammatory pseudotumor, and sclerosing pancreatitis-associated sclerosing cholangitis: do they belong to a spectrum of sclerosing pancreatitis? Am J Surg Pathol 28: 1193-1203, 2004.

5. Hamano H, Kawa S, Ochi $\mathrm{Y}$, et al. Hydronephrosis associated with retroperitoneal fibrosis and sclerosing pancreatitis. Lancet 359: 1403-1404, 2002.

6. Taniguchi $\mathrm{T}$, Ko M, Seko $\mathrm{S}$, et al. Interstitial pneumonia associated with autoimmune pancreatitis. Gut 53: 770, 2004.

(C) 2008 The Japanese Society of Internal Medicine http://www.naika.or.jp/imindex.html 